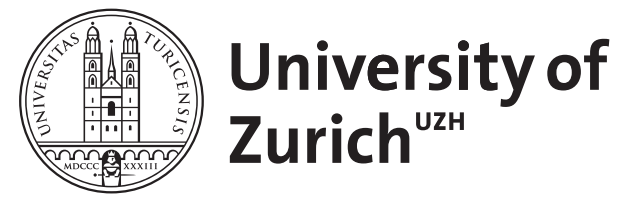

\title{
Blutdruckkontrolle bei Patienten mit polyzystischer Nierenerkrankung
}

Wüthrich, R P ; Kistler, A D

DOI: https://doi.org/10.1007/s11560-014-0944-3

Posted at the Zurich Open Repository and Archive, University of Zurich

ZORA URL: https://doi.org/10.5167/uzh-122754

Journal Article

Accepted Version

Originally published at:

Wüthrich, R P; Kistler, A D (2015). Blutdruckkontrolle bei Patienten mit polyzystischer Nierenerkrankung. Der Nephrologe, 10:201-206.

DOI: https://doi.org/10.1007/s11560-014-0944-3 
Manuskript „Blutdruckkontrolle bei Patienten mit polyzystischer Nierenerkrankung” für die Zeitschrift Der Nephrologe $3 / 2015$

\section{DOI 10.1007/s11560-014-0944-3}

Korrespondenzadresse: Prof. Dr. R.P. Wüthrich

Klinik für Nephrologie, Universitätsspital

Rämistr. 100, CH-8091 Zürich, Schweiz

rudolf.wuethrich@usz.ch

Weitere Autoren: $\quad$ A.D. Kistler

$\checkmark \quad$ Bitte kreuzen Sie die gewünschten Optionen an und senden Sie dieses Formular an die oben angegebene E-Mail-Adresse oder Faxnummer. Dies ist auch dann wichtig, wenn Sie keine Korrekturen haben. Vielen Dank. Die Erteilung der Rechtseinräumung mit Ihrer Unterschrift ist für eine Veröffentlichung erforderlich!

\section{Imprimatur}

Bitte beachten Sie, dass nach Imprimatur keine weiteren Textkorrekturen, Änderungen der Autorenschaft oder der

Autorenreihenfolge möglich sind.

Folgende Korrekturmöglichkeiten stehen Ihnen offen:

- Fügen Sie Ihre Korrekturen komfortabel mit der Kommentarfunktion Ihres PDF-Programms (z.B. Adobe Professional)

direkt ins Beitrags-PDF ein.

- Alternativ können Sie das PDF ausdrucken und Ihre Korrekturen gut lesbar in den Ausdruck einfügen.

Hiermit gebe ich mein Manuskript direkt frei. Korrekturen sind nicht notwendig.

Ich habe einzelne Seiten des Manuskriptes korrigiert und diese der Antwort beigefügt. Nach ausgeführter Korrektur ist das Manuskript imprimiert.

Folgende Manuskript-Seiten habe ich beigefügt:

$\square \quad$ Diese Rückantwort enthält das komplette Manuskript mit entsprechenden Korrekturwünschen. Nach ausgeführter Korrektur ist das Manuskript imprimiert. Mir ist bewusst, dass größere Korrekturen oder Änderungen an der Textlänge ein späteres Publikationsdatum nach sich ziehen können.

\section{Sonderdrucke}

Ich wünsche kostenpflichtige Sonderdrucke meines veröffentlichten Manuskriptes. Das ausgefüllte Formular „Sonderdruckbestellung“ liegt bei.

\section{OpenAccess}

Springer bietet Ihnen die Möglichkeit, Ihren Artikel Open Access über das Springer Open Choice Programm zu veröffentlichen. Ihr Beitrag wird dann sofort und dauerhaft für jedermann weltweit online freiverfügbar. Dies ist der einfachste Weg für Sie Open Access Mandaten zu entsprechen, da der Beitrag unter der liberalen Creative Commons Attribution (CC BY) Lizenz publiziert wird, welche die freie Distribution und Weiternutzung der finalen Version unterstützt. Die einmalige Open Access Gebühr beträgt EUR 2.200 (netto).

Ich wünsche nähere Informationen über die Möglichkeit, mein Manuskript Open Access zu publizieren.

\section{Einhaltung ethischer Richtlinien}

\section{IVa Interessenkonflikt}

Die Angabe eines Interessenkonflikts ist seriöse Publikationspraxis. Bitte geben Sie alle finanziellen oder persönlichen Beziehungen von Ihnen und Ihren Koautoren zu Dritten an, deren Interessen vom Beitragsinhalt positiv oder negativ betroffen sein können, auch wenn aus Ihrer Sicht keine Beeinflussung stattgefunden hat. Beispiele: finanzielle Verbindung zu Pharmafirmen, Beschäftigungsverhältnisse, Beratungs- und Referententätigkeiten, Honorare, Reisekostenübernahmen, Drittmittel, Aktienbesitz. Die Erklärung bezieht sich auf die Gegenwart und die vergangenen 5 Jahre und wird veröffentlicht.

Auch wenn kein Interessenkonflikt besteht, ist dies anzugeben. Sollte der Interessenkonflikt bereits im Manuskript genannt worden sein, erübrigt sich die Angabe an dieser Stelle.

Der korrespondierende Autor gibt für sich und seine Koautoren an, dass kein Interessenkonflikt besteht.

Der korrespondierende Autor weist für sich und seine Koautoren auf folgende Beziehung/en hin (Bitte machen Sie separate Angaben zu jedem Autor auch wenn nicht für jeden Autor ein Interessenkonflikt besteht): 
IVb Patientenrechte und Tierschutzbestimmungen

Medizinische Forschung unterliegt ethischen Standards, die die Achtung vor den Menschen fördern und ihre Gesundheit und Rechte schützen. Ärzte sollen die ethischen, rechtlichen und behördlichen Normen und Standards für Forschung am Menschen und am Tier ihrer eigenen Länder sowie die maßgeblichen internationalen Normen und Standards berücksichtigen.

Bitte machen Sie im Folgenden die für das vorliegende Manuskript zutreffenden Angaben.

$\square \quad$ Hiermit bestätige ich als korrespondierender Autor, dass das vorliegende Manuskript keine Studien an Menschen oder Tieren enthält.

Falls Patienten durch Abbildungen oder anderweitige Angaben im Manuskript zu identifizieren sind:

$\square \quad$ Alle Patienten, die über Bildmaterial oder anderweitige Angaben innerhalb des Manuskripts zu identifizieren sind, haben hierzu ihre schriftliche Einwilligung gegeben. Im Falle von nicht mündigen Patienten liegt die Einwilligung eines Erziehungsberechtigten oder des gesetzlich bestellten Betreuers vor.

Falls dem vorliegenden Manuskript Studien am Menschen zugrunde liegen:

$\square \quad$ Alle im vorliegenden Manuskript beschriebenen Untersuchungen am Menschen wurden mit Zustimmung der zuständigen Ethik-Kommission, im Einklang mit nationalem Recht sowie gemäß der Deklaration von Helsinki von 1975 (in der aktuellen, überarbeiteten Fassung) durchgeführt. Von allen beteiligten Patienten liegt eine Einverständniserklärung vor.

Falls dem vorliegenden Manuskript Experimente mit Labortieren zugrunde liegen:

$\square \quad$ Hiermit bestätige ich als korrespondierender Autor, dass alle nationalen Richtlinien zur Haltung und zum Umgang mit Labortieren eingehalten wurden und die notwendigen Zustimmungen der zuständigen Behörden vorliegen.

\section{Rechtseinräumung}

In Erweiterung des §38 Abs. 1 UrhG räume ich als erstgenannter Verfasser der Springer Science+Business Media Gruppe das ausschließliche, geografisch und zeitlich unbeschränkte Recht der Speicherung, Vervielfältigung, Verbreitung und Wiedergabe meines Beitrages (inkl. Bilder, Video- und Audiodateien) ein. Dies schließt das Recht zur Übersetzung und Bearbeitung ein und gilt für die Dauer des gesetzlichen Urheberrechts für alle Auflagen und Publikationen in gedruckter und elektronischer Form (z.B. online, offline, mobile Nutzung, embedded content). Springer ist berechtigt, die Nutzungsrechte am gesamten Beitrag und Teilen daraus in gedruckter und elektronischer Form wahrzunehmen und weiterzugeben.

Hiermit versichere ich - auch im Namen der Miturheber -, dass ich berechtigt bin, über die urheberrechtlichen Nutzungsrechte an dem o.g. Beitrag zu verfügen. 


\section{Autorenfortdrucke - Bestellung}

Wenn Sie Fortdrucke Ihres Artikels bestellen möchten, füllen Sie bitte dieses Formular aus und senden es zusammen mit Ihren Korrekturen an uns zurück. Bitte beachten Sie, dass alle Anzeigen, die in der gedruckten Version Ihres Beitrags im Heft veröffentlicht werden, auch im Sonderdruck erscheinen. Die Lieferzeit für Fortdrucke beträgt produktionsbedingt 3 bis 6 Wochen.

Sollten Sie erst nach dem Druck eines Heftes Fortdrucke bestellen, beträgt die Mindestabnahme 100 Exemplare, da hier ein erhöhter Aufwand zu berücksichtigen ist. Diese Sonderdrucke werden als kommerzielle Sonderdrucke kalkuliert.

Achtung: Ihre Bestellung kann nur bearbeitet werden, wenn eine Kreditkartennummer und Unterschrift vorliegen.

Hiermit bestelle ich:

\begin{tabular}{|l:l|}
\hline$\square$ 50 Exemplare & 300 EUR \\
\hline$\square \mathbf{1 0 0}$ Exemplare & 365 EUR \\
\hline$\square \mathbf{2 0 0}$ Exemplare & 525 EUR \\
\hline$\square \mathbf{3 0 0}$ Exemplare & 680 EUR \\
\hline (Preise inkl. Porto und Verpackung, zzgl. MwSt.)
\end{tabular}

Für die Bestellung von mehr als 300 Exemplaren, oder von Sonderdrucken, die nach Druck des Heftes produziert werden, wenden Sie sich bitte an:

Kontakt Reprints

Christoph Holzhaus

Telefon 030 / 827 87-57 14, Fax -6 5714

E-Mail christoph.holzhaus@springer.com

Bitte geben Sie Ihre VAT-Nummer an:

Kunden aus EU-Ländern ohne VAT-Nummer müssen den allgemein gültigen Steuerbetrag addieren.

Bitte geben Sie Ihre Kreditkartennummer an:

$\square$ Eurocard/Access/Mastercard

$\square \quad$ American Express

$\square \quad$ Visa/Barclaycard/BankAmericard

Kartennummer (inkl. Prüfziffern):

Gültig bis: _ _ _ _

Datum / Unterschrift:
Rechnungsanschrift:

$\square \quad$ siehe Lieferanschrift

$\square$

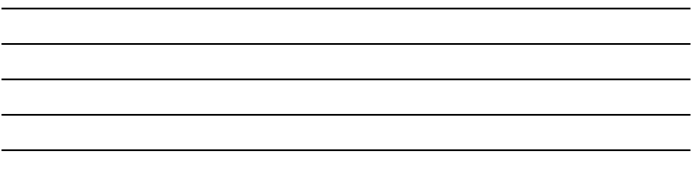

Für Autoren wohnhaft in Deutschland ist die Zahlung per Bankeinzug möglich:

Hiermit erteile ich Springer die

Abbuchungsgenehmigung von meinem Bankkonto über den Rechnungsbetrag bei Fälligkeit.

Kontonr.:

Bankleitzahl:

Bank:

Datum / Unterschrift:

Bitte verwenden Sie bei der Rechnungsstellung folgende Bestellnummer/folgendes Zeichen:

Bitte überprüfen Sie die oben angegebenen Daten und korrigieren Sie sie gegebenfalls. 
- IV - 


\begin{tabular}{|l|l|}
\hline DOI & $10.1007 /$ s $11560-014-0944-3$ \\
\hline Copyright & Springer-Verlag Berlin Heidelberg - 2015 \\
\hline
\end{tabular}

\section{Leitthema}

\section{Blutdruckkontrolle bei Patienten mit polyzystischer Nierenerkrankung}

R.P. Wüthrich · A.D. Kistler

Klinik für Nephrologie, Universitätsspital, Zürich, Schweiz

\section{Korrespondenzadresse}

Prof. Dr. R.P. Wüthrich

Klinik für Nephrologie, Universitätsspital

Rämistr. 100

$\mathrm{CH}-8091$ Zürich

Schweiz

rudolf.wuethrich@usz.ch

\section{Prof. Dr. R.P. Wüthrich}

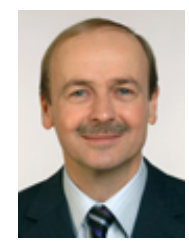

Online publiziert: Onlinedatum erscheint nach Freigabe

\section{Zusammenfassung}

Hintergrund Die autosomal-dominante polyzystische Nierenerkrankung (,,autosomal dominant polycystic kidney disease“, ADPKD) ist die häufigste genetische Nierenerkrankung und betrifft weltweit 6-12 Mio. Menschen. Die Erkrankung ist durch eine progressive Bildung von unzähligen Nierenzysten charakterisiert, welche das normale Nierengewebe sukzessive verdrängen. In der Folge kommt es zum Verlust der Nierenfunktion und 
zur Dialysepflichtigkeit ab der 5. Lebensdekade. Es gibt noch keine kausale Therapie, welche die Krankheitsprogression signifikant verlangsamen oder stoppen würde. Bei den meisten Patienten mit ADPKD entwickelt sich eine arterielle Hypertonie. Diese entsteht relativ früh im Krankheitsverlauf und ist hauptsächlich durch Aktivierung des Renin-Angiotensin-Aldosteron-Systems (RAAS) und des Sympathikus im Rahmen der renalen Zystenbildung bedingt. Die Hypertonie ist mitverantwortlich für die gesteigerte kardiovaskuläre Morbidität und Mortalität von Patienten mit Zystennieren. Eine optimale Einstellung des Blutdrucks ist wichtig, um die Prognose der Zystenerkrankung und der assoziierten Herz-Gefäß-Erkrankungen zu verbessern.

Schlussfolgerung Zielblutdruckwerte und Wahl von geeigneten Antihypertensiva bei Patienten mit ADPKD konnten noch nicht abschließend in Leitlinien festgelegt werden. Die vor Kurzem publizierten Resultate der HALT-PKD-Studien legen nahe, dass ein Zielblutdruck unter 130/80 mmHg mittels RAAS-Blockade bei Patienten mit ADPKD angestrebt werden sollte.

\section{Schlüsselwörter}

Hypertonie · Polyzystische Nierenerkrankung · Renin-Angiotensin-Aldosteron-System · Sympathikus $\cdot$ Herz-Gefäß-Erkrankungen

\section{Blood pressure control in patients with polycystic kidney disease}

\section{Abstract}

Background Autosomal dominant polycystic kidney disease (ADPKD) is the most prevalent genetic kidney disease and affects 6-12 million people worldwide. The disease is characterized by the progressive development of innumerable renal cysts that gradually replace normal kidney tissue, ultimately leading to the loss of renal function starting from the fifth decade of life. There is no causal therapy which significantly slows or stops disease progression. Most patients with ADPKD develop hypertension. High blood pressure develops early in the course of the disease and is mainly caused by activation of the renin-angiotensin-aldosterone system (RAAS) and the sympathetic nervous system. Hypertension is jointly responsible for the increased cardiovascular morbidity and mortality 
in patients with ADPKD. The optimal therapy of hypertension is essential to improve the prognosis of the cystic disease and the associated cardiovascular diseases.

Conclusions Target blood pressures and selection of suitable antihypertensive drugs for patients with ADPKD have not been definitively defined in guidelines. The recently published results from the HALT-PKD studies suggest that a target blood pressure of $<130 / 80 \mathrm{mmHg}$ by inhibition of the RAAS should be aimed for in patients with ADPKD.

\section{Keywords}

Hypertension $\cdot$ Polycystic kidney disease $\cdot$ Renin-angiotensin system $\cdot$ Sympathetic nervous system $\cdot$ Cardiovascular diseases

\section{Klinische Charakteristika der ADPKD}

Die autosomal dominante polyzystische Nierenerkrankung (ADPKD) ist eine der häufigsten monogenetischen Erkrankungen des Menschen und ist Ursache eines terminalen Nierenversagens bei 7-10\% der Dialysepatienten [28]. Mutationen in den Genen PKD1 (85\% der Fälle; klinisch schwerere Form) und PKD2 (15\% der Fälle; klinisch langsamer progrediente Form) sind ursächlich für die Erkrankung verantwortlich [10]. Die ADPKD manifestiert sich klinisch meist im Alter zwischen 25 und 45 Jahren. Die häufigsten Erstmanifestationen beinhalten

1. arterielle Hypertonie schon in jüngerem Alter,

2. Abdominal-/Flankenschmerzen (gelegentlich im Rahmen einer Zystenblutung oder eines Zysteninfekts),

3. Hämaturie (meist Mikro-, aber auch Makrohämaturie bei Zystenblutungen),

4. mäßiggradige Polyurie (manifestiert sich auch als Nykturie).

Die Progression der ADPKD zeigt eine große interindividuelle Variabilität [21].

Die Erkrankung ist durch eine zunehmende Bildung und Expansion von

flüssigkeitsgefüllten Zysten im Parenchym beider Nieren gekennzeichnet. Im Verlauf kommt es zur fortschreitenden Vergrößerung der Zysten durch Proliferation des Zystenepithels und Sekretion von Zystenflüssigkeit. Dadurch wird das umliegende Nierengewebe komprimiert, 
und es kommt zur Reduktion der glomerulären Filtrationsrate (GFR). Bei Beginn der Dialysepflichtigkeit sind die Nieren massiv vergrößert und komplett von Zysten durchsetzt, die von fibrotischen Arealen und atrophischen Tubuli umgeben sind.

Die ADPKD zeigt nebst dem genannten renalen Befall verschiedene extrarenale Manifestationen:

- Zystenbildung in Leber und Pankreas;

- Kolondivertikel und Bauchwandhernien;

- Mitralklappenprolaps (selten eine Mitralinsuffizienz);

- Aortenklappenanomalien;

- die gefürchteten intrakraniellen Aneurysmen, die zu Ruptur und potenziell letaler Subarachnoidalblutung führen können, letztere tritt familiär gehäuft auf, ist insgesamt aber selten [19].

\section{Arterielle Hypertonie bei ADPKD}

\section{Allgemeine Aspekte}

Die arterielle Hypertonie tritt bei Patienten mit ADPKD relativ früh im Krankheitsverlauf auf, in der Regel wesentlich früher als in der Normalbevölkerung [ $[\underline{8}, \underline{14}]$. Das mediane Alter bei Diagnosestellung der Hypertonie liegt bei 32 Jahren bei Männern und 34 Jahren bei Frauen [26]. Bei $P K D 1$-Mutationen tritt eine behandlungsbedürftige Hypertonie etwa 5 Jahre früher auf als bei PKD2-Mutationen, und bei 50-70\% der Patienten findet man eine Hypertonie, noch bevor die Nierenfunktion eingeschränkt ist [4]. Das Ausmaß der Hypertonie korreliert mit dem Volumen der Zysten in den Nieren und mit der Wachstumsgeschwindigkeit der Zysten [7]. Als Folge der arteriellen Hypertonie findet sich bei Patienten mit ADPKD schon früh im Krankheitsverlauf eine linksventrikuläre Hypertrophie (LVH). Bekannterweise sind Hypertonie und LVH mit einem gesteigerten kardiovaskulären Risiko assoziiert, so auch bei Patienten mit ADPKD. Die Hypertonie stellt vermutlich die am besten behandelbare Variable bei Patienten mit ADPKD dar. Deshalb sind die frühe Diagnosestellung und eine optimale Behandlung von großer Bedeutung, um das Zystenwachstum zu verlangsamen und die kardiovaskulären Komplikationen zu verhindern [2, $\underline{3}]$. 


\section{Pathophysiologie der Hypertonie bei ADPKD}

Die arterielle Hypertonie tritt bei ADPKD bei noch normaler exkretorischer Nierenfunktion auf und lässt sich daher nicht allein auf eine gestörte Salzexkretion und andere unspezifische Mechanismen der renalen Hypertonie zurückführen. Vielmehr scheinen spezifische Mechanismen für die Hypertonie bei ADPKD verantwortlich zu sein (Abb. 1; [르, 24]). Eine zentrale Bedeutung kommt dabei dem Renin-Angiotensin-Aldosteron-System (RAAS) zu: Durch die Zystenbildung entsteht eine lokale Ischämie, was zur Aktivierung des intrarenalen RAAS führt. Dies konnte in zahlreichen experimentellen und klinischen Studien belegt werden [20]. Zusätzlich konnte gezeigt werden, dass bei ADPKD eine Hyperaktivität des sympathischen Nervensystems entsteht, welche zur Pathogenese der Hypertonie beiträgt [16]. Ferner ist bekannt, dass durch den renalen Konzentrierungsdefekt und die konsekutive Neigung zu Polyurie eine latente Stimulation von Vasopressin über deren vaskuläre $V_{1}$-Rezeptoren sowie eine Vasokonstriktion und eine konsekutive Blutdrucksteigerung entstehen. Schließlich werden Polycystin 1 und 2 auch in Endothelzellen und Gefäßmuskelzellen exprimiert, wo sie über Endothelin und Stickoxid (NO) bzw. über die intrazelluläre Kalziumhomöostase an der Regulation des Gefäßtonus beteiligt sind. So scheint es sich bei der arteriellen Hypertonie bei ADPKD teilweise auch um eine extrarenale (vaskuläre) Manifestation zu handeln [ㄴ, 20].

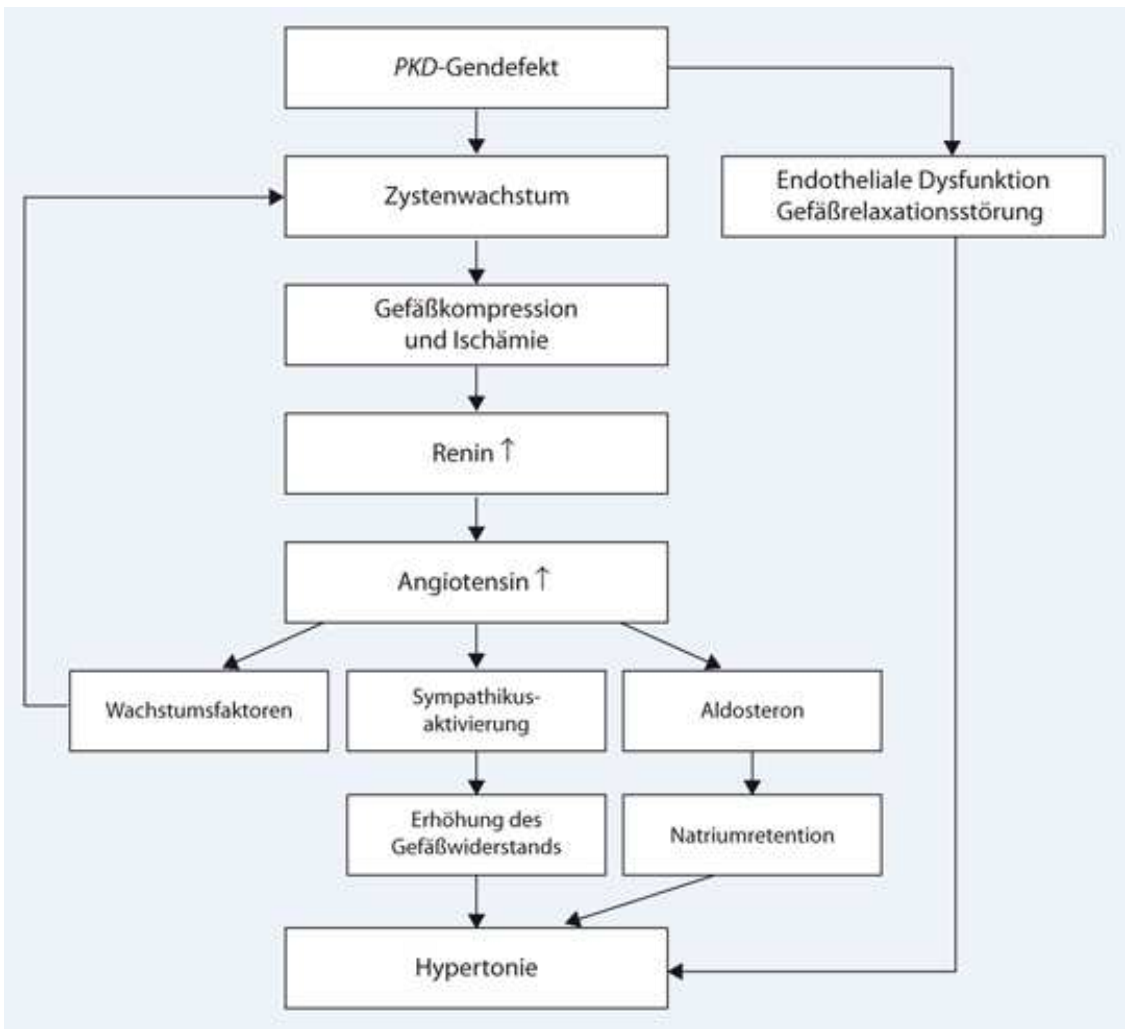


Abb. 1 Pathogenese der Hypertonie bei autosomal dominanter polyzystischer Nierenerkrankung (ADPKD)

\section{Behandlung der Hypertonie bei ADPKD}

\section{Allgemeine Aspekte}

Die frühzeitige, wirkungsvolle und langjährige Behandlung der Hypertonie ist entscheidend im Management von Patienten mit ADPKD [23]. Die Entwicklung einer Hypertonie ist gleichzusetzen mit einer Progression der Zystenerkrankung. Es wurde empfohlen, mit medikamentöser Therapie Zielwerte bei Erwachsenen von unter 130/80 mmHg und bei Kindern Werte unterhalb der 75. Perzentile anzustreben [9]. Die Ziele der antihypertensiven Therapie bestehen in einer Reduktion einerseits der extrarenalen hypertensiven Folgeerkrankungen (LVH, Arteriosklerose) und andererseits der hypertensiven Nierenschädigung (Nephroangiosklerose, Glomerulosklerose). Zusätzlich gibt es Hinweise, dass die Hypertonie bzw. die intrarenale RAAS-Aktivierung ursächlich das Zystenwachstum begünstigen kann: So konnten ACE (,,angiotensine-converting enzyme“)-Hemmer in tierexperimentellen Studien, möglicherweise durch eine Antagonisierung des mitogenen Effekts des RAAS, das Zystenwachstum hemmen [13, 30].

\section{$>$ Die frühzeitige, wirkungsvolle und langjährige Behandlung der Hypertonie ist entscheidend im Management von Patienten mit ADPKD}

Klinisch stellen sich bezüglich der Hypertoniebehandlung bei ADPKD entsprechend folgende drei Fragen:

1. Welche Medikamentenklassen (bzw. nichtmedikamentösen Therapien) sind zu bevorzugen?

2. Welches sind die optimalen Blutdruckzielwerte bzw. ab welchem Blutdruck soll eine medikamentöse Therapie erfolgen?

3. Haben ACE-Hemmer/Angiotensinrezeptorblocker (ARB) unabhängig vom Blutdruck einen protektiven Effekt?

\section{Therapeutische Optionen zur Behandlung der Hypertonie bei ADPKD}

Bei Patienten mit ADPKD gelten zunächst die üblichen Grundregeln der nichtmedikamentösen Therapie der Hypertonie (Einschränkung der Zufuhr von Kochsalz und Koffein, Bewegung, Rauchstopp). In der Regel ist der Blutdruck bei Patienten 
mit ADPKD mit Medikamenten relativ leicht einzustellen, resistente Formen der Hypertonie sind sehr selten. Oft genügt ein Medikament mit einem einzelnen Wirkstoff oder ein Kombinationspräparat mit einem Thiaziddiuretikum. Aufgrund der genannten pathophysiologischen Überlegungen würden RAAS-Inhibitoren (ACE-Hemmer und ARB) die erste Wahl darstellen. Wenngleich bisher keine ausreichend großen und methodisch konklusiven Studien existierten, welche die Überlegenheit von RAAS-Inhibitoren belegten, gab es doch viele indirekte Hinweise, dass diese Substanzklassen zu bevorzugen sind. Folgende kleinere Studien sind erwähnenswert:

- In einer nichtrandomisierten Studie bei hypertensiven ADPKD-Patienten nahm die GFR unter Diuretika schneller ab als unter ACE Hemmern []].

- In einer prospektiven randomisierten Studie mit 24 Patienten wurde die Wirkung von Amlodipin und Enalapril auf Blutdruck, Proteinurie und GFR über 5 Jahre untersucht. Bei vergleichbarer Blutdruckkontrolle reduzierte nur Enalapril die Proteinurie, beide Gruppen zeigten aber einen vergleichbaren Rückgang der GFR [ [5].

- In einer weiteren kleinen prospektiven Studie wurden 49 Patienten randomisiert und mit Amlodipin oder Candesartan während 3 Jahren behandelt. Bei vergleichbarer Blutdruckkontrolle beobachtete man, dass unter Amlodipin 24\% der Patienten eine Verdoppelung des Serumkreatinins zeigten gegenüber nur 4,2\% unter Candesartan [18].

- Eine kleine und nichtkonklusive 2-jährige Studie verglich bei 26 Patienten den Effekt von Kalziumantagonisten mit ACE-Hemmern und fand keinen Unterschied bezüglich Blutdruckkontrolle oder Serumkreatinin [12].

- Eine retrospektive Studie mit 32 Patienten dokumentierte ebenfalls einen größeren Verlust der GFR unter Kalziumantagonisten als unter RAAS-Hemmung mit ACE-Hemmer oder ARB [17].

- Eine Studie verglich den ACE-Hemmer Enalapril mit dem Betablocker Atenolol oder Placebo und fand keinen Unterschied bezüglich GFR-Verlust [29].

- Eine Studie an 85 Kindern mit ADPKD zeigte, dass Enalapril die Nierenfunktion und die LVH stabilisieren konnte, dass aber das Nierenvolumen weiterhin zunahm [1]].

- Schließlich zeigte eine Metaanalyse von 11 randomisierten Studien, welche 1860 Patienten mit nichtdiabetischen Nephropathien und darunter 142 Patienten mit ADPKD einschlossen, dass ACE-Hemmer gegenüber anderen Blutdruckmedikamenten effektiver waren bezüglich Senkung der Proteinurie bei Patienten mit fortgeschrittener ADPKD, speziell 
bei Patienten mit stärkerer Proteinurie. Der Effekt der ACE-Hemmer auf das Fortschreiten der Niereninsuffizienz war jedoch nicht konklusiv und die ADPKD-Population insofern atypisch, als dass sich eine deutlich höhere Proteinurie zeigte, als bei ADPKD typisch ist [11].

Zusammenfassend gibt es keine eindeutige Evidenz für eine Überlegenheit von RAAS-Blockern bezüglich klinisch relevanter Endpunkte, wobei aber sämtliche erwähnten Studien zu klein und von zu kurzer Dauer waren, um konklusive Ergebnisse zu produzieren. Aus diesen kleineren und zum Teil methodisch fraglichen Studien gibt es jedoch Hinweise, dass die Hemmung des RAAS mit ACE-Hemmern oder ARB eine gute und sichere Blutdruckkontrolle ermöglicht und sich auf gewisse Endpunkte besser auswirkt als andere Medikamente, insbesondere Kalziumantagonisten.

\section{Blutdruckzielwerte bei ADPKD}

Krankheitsspezifische Blutdruckzielwerte wurden für die ADPKD bisher noch nicht abschließend definiert. Eine Subgruppenanalyse der MDRD-Studie, unter deren Teilnehmern sich 200 Patienten mit ADPKD befanden, zeigte bei Patienten mit einer GFR von $25-55 \mathrm{ml} / \mathrm{min} / 1,73 \mathrm{~m}^{2}$ keinen Unterschied zwischen normaler (MAP-Ziel $\leq 113 \mathrm{mmHg}$ bei über 60-Jährigen und $\leq 107$ bei unter 60 -Jährigen) und strikter (MAP-Ziel $\leq 92 \mathrm{mmHg}$ bei über 60-Jährigen und $\leq 88$ bei unter 60-Jährigen) Blutdruckkontrolle. Bei Patienten mit einer GFR von 13-24 ml/min/1,73 $\mathrm{m}^{2}$ zeigte sich jedoch eine etwas raschere GFR-Abnahme in der Gruppe mit strikter Blutdruckkontrolle [15]. Eine weitere randomisiert kontrollierte Studie mit 75 ADPKD-Patienten fand bei einer strikten $(<120 / 80 \mathrm{mmHg})$ gegenüber einer normalen (135-140/85-90 mmHg) Blutruckkontrolle mittels Enalapril oder Amlodipin über 7 Jahre keinen Unterschied bezüglich der Nierenfunktion, jedoch einen signifikant positiven Effekt auf die LVH [22].

\section{$>$ Krankheitsspezifische Blutdruckzielwerte wurden für die ADPKD bisher noch nicht abschließend definiert}

Die Frage nach Blutdruckzielwerten und die Frage, ob die Progression der Nierenerkrankung (Zystenwachstum und GFR-Verlust) durch eine RAAS-Blockade gehemmt werden kann, waren Gegenstand des HALT-PKD-Studienprogramms, dessen Daten nun im November 2014 an der Jahrestagung der American Society of Nephrology vorgestellt und im New England 
Journal of Medicine publiziert wurden [25, 27]. Diese Daten sind vor allem deshalb sehr wertvoll, weil sie die mit Abstand größten und methodisch gut durchgeführten Studien zur Hypertoniebehandlung bei ADPKD darstellen.

\section{HALT-PKD-Resultate}

Das in den Jahren 2006 bis 2014 durchgeführte HALT-PKD-Studienprogramm untersuchte den Effekt der RAAS-Blockade und der strengen Kontrolle des Blutdrucks auf die Krankheitsprogression bei erwachsenen Patienten mit ADPKD (Abb. 2). Diese Studien aufgeteilt in die Teilstudien A und B - studierten die Wirkung der RAAS-Blockade mit dem ACE-Hemmer Lisinopril und dem ARB Telmisartan auf das Fortschreiten der Krankheit bei Patienten mit erhaltener GFR (Studie A; GFR $>60 \mathrm{ml} / \mathrm{min} / 1,73 \mathrm{~m}^{2} ; \mathrm{n}=558$ ) und bei Patienten mit fortgeschrittener Nierenerkrankung (Studie B; GFR 25-60 ml/min/1,73 m²; n=486).

In Studie A wurden die Patienten in einem 2×2-faktoriellen Studiendesign in die Gruppen [Lisinopril + Placebo] versus [Lisinopril + Telmisartan] und in der zweiten Ebene auf einen normalen $(120 / 70$ bis $130 / 80 \mathrm{mmHg})$ versus tiefen Zielblutdruck $(95 / 60$ bis 110/75 mmHg) randomisiert. In Studie B wurden [Lisinopril + Placebo] versus [Lisinopril + Telmisartan] bei gleichem Blutdruckzielwert (110-130/70-80 mmHg) verglichen. Primäre Endpunkte waren in Studie A das Nierenvolumen und in Studie B ein Kompositendpunkt aus Halbierung der GFR-Verschlechterung, Nierenversagen oder Tod.

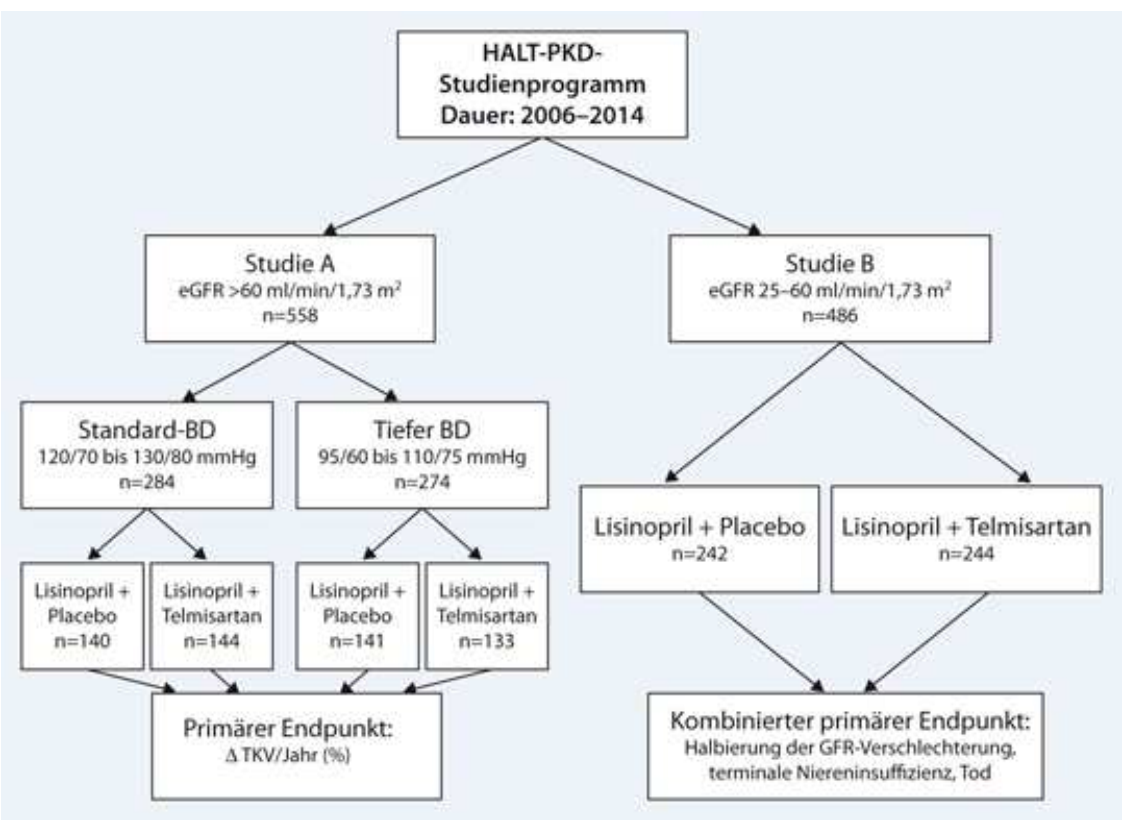

Abb. 2 Schematische Darstellung des HALT-PKD-Studienprogramms, mit den eGFR (geschätzte glomeruläre Filtrationsrate)-Kriterien für den Einschluss in Studie A (erhaltene GFR) und B (reduzierte 
GFR). Studie A hat ein 2×2-faktorielles Design (Standard- vs. tiefer Blutdruck $(B D)$ sowie Vergleich Lisinopril/Placebo vs. Lisinopril/Telmisartan)

In Studie A zeigten Patienten mit tiefer eingestelltem Blutdruck ein geringeres Volumenwachstum der Nieren (5,6 vs 6,6\%, p=0,006; Abb. 3), die Studie war bezüglich des primären Endpunkts also positiv, wenngleich der Effekt gering war. Es zeigten sich keine Unterschiede bezüglich der Nierenfunktionsverschlechterung (jährlicher GFR-Verlust -2,9 vs. $-3,0 \mathrm{ml} / \mathrm{min} / 1,73 \mathrm{~m}^{2}$; Tab. 1). Ferner zeigte sich in der Behandlungsgruppe mit tieferem Blutdruck eine stärkere Reduktion des linksventrikulären Massenindexes (LVMI) und der Albuminurie, jedoch kam es häufiger zu orthostatischen Beschwerden (Schwindel, Taumel). Beim Vergleich [Lisinopril + Placebo] versus [Lisinopril + Telmisartan] zeigte sich kein Unterschied bezüglich des Volumenwachstums der Nieren (Abb. 3), des GFR-Verlusts, der Albuminurie und des LVMI.

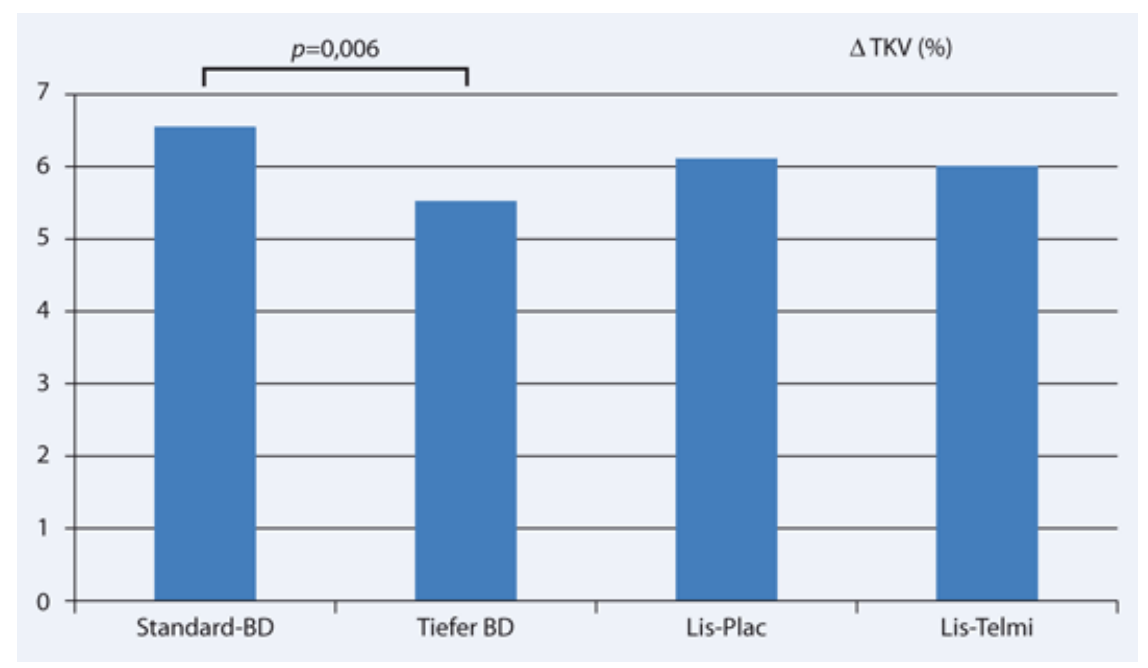

Abb. 3 Jährliche Veränderung des totalen Nierenvolumens ( $\triangle T K V)$ in Prozent in der HALT-PKD-Studie A: Die tiefere Einstellung des Blutdrucks $(B D)$ war mit einem geringeren Wachstum vergesellschaftet (5,6 vs. $6,6 \%$, relativer Unterschied: $14,2 \%, p=0,006)$, wogegen die Zugabe von Temisartan (Telmi) vs. Placebo (Plac) zu Lisinopril (Lis) das Volumenwachstum nicht signifikant veränderte $(6,0$ vs $6,2 \%, p=0,52)$

In Studie B zeigte sich beim Vergleich [Lisinopril + Placebo] versus [Lisinopril + Telmisartan] bei Patienten mit weiter fortgeschrittener Zystenerkrankung als in Studie A (höheres Alter, schlechtere GFR) kein Unterschied bezüglich des kombinierten Endpunkts (Halbierung der GFR-Verschlechterung, Erreichen einer ESRD oder Tod), und es fand sich auch kein Unterschied bezüglich GFR-Verlust und Albuminurie. Unter Doppeltherapie mit 
Lisinopril und Telmisartan wurden auch nicht mehr Fälle mit Hyperkaliämie und akutem Nierenversagen festgestellt.

Zusammenfassend schien der tiefere Blutdruck im früheren Stadium der Erkrankung den Krankheitsverlauf günstig zu beeinflussen, jedoch auf Kosten von vermehrten hypotensiven Nebenwirkungen. Die Doppelblockade zeigte gegenüber einer Monotherapie mit ACE-Hemmer weder bei Patienten mit früher noch bei solchen mit fortgeschrittener Erkrankung einen stärkeren Effekt, zeigte aber auch keine erhöhte Komplikationsrate, wie dies in anderen Studien zur RAAS-Doppelblockade bei Diabetikern aufgefallen war.

Kritisch muss angemerkt werden, dass in beiden Studien die Dosis des ACE-Hemmers in der Gruppe mit Doppelblockade geringerer war als in der Vergleichsgruppe. Ferner war der Blutdruck in beiden Gruppen vergleichbar, und es fand sich kein Unterschied in der Suppression der Urinaldosteronwerte. Daraus kann gefolgert werden, dass die Suppression des RAAS in beiden Therapiegruppen vergleichbar gewesen war bzw. dass die Kombination von ACE-Hemmer und ARB nicht wirksamer war als die Monotherapie mit einem ACE-Hemmer in höherer Dosierung. Leider gab es in den HALT-PKD-Studien keinen Kontrollarm ohne Hemmer des RAAS, weshalb letztendlich unklar bleibt, ob die Hemmung des RAAS gegenüber einer Blutdruckeinstellung mit z. B. Betablocker und/oder Diuretika überlegen ist oder nicht. Immerhin scheint die niedrigere Blutdruckeinstellung das Nierenwachstum zu hemmen; aber auch hier konnte nicht gezeigt werden, dass mit dieser Strategie die Progression des GFR-Verlusts bis hin zur terminalen Niereninsuffizienz verzögert werden kann.

\section{Fazit für die Praxis}

- Patienten mit ADPKD entwickeln früh im Krankheitsverlauf eine Hypertonie, die zur Krankheitsprogression beiträgt und das kardiovaskuläre Risiko erhöht. Die Hypertonie ist in der Regel medikamentös gut einstellbar, resistente Formen sind sehr selten.

- Wegen der ausgeprägten intrarenalen Aktivierung des RAAS sind ACE-Hemmer oder ARB, ggf. in Kombination mit einem niedrig dosierten Thiaziddiuretikum, bevorzugte Medikamente.

- Die Zielblutdruckwerte sollten unter 130/80 mmHg liegen, eine stärkere Blutdrucksenkung kann aufgrund der neuen Daten aus den HALT-PKD-Studien empfohlen werden, sofern dies von den Nebenwirkungen her vertretbar ist. 
Einhaltung ethischer Richtlinien Interessenkonflikt. R.P. Wüthrich und A. Kistler geben an, dass kein Interessenkonflikt besteht.

Dieser Beitrag beinhaltet keine Studien an Menschen oder Tieren.

\section{Literatur}

1. Cadnapaphornchai MA, Mcfann K, Strain JD et al (2009) Prospective change in renal volume and function in children with ADPKD. Clin J Am Soc Nephrol 4:820-829

2. Chapman AB, Johnson AM, Rainguet S et al (1997) Left ventricular hypertrophy in autosomal dominant polycystic kidney disease. J Am Soc Nephrol 8:1292-1297

3. Chapman AB, Schrier RW (1991) Pathogenesis of hypertension in autosomal dominant polycystic kidney disease. Semin Nephrol 11:653-660

4. Chapman AB, Stepniakowski K, Rahbari-Oskoui F (2010) Hypertension in autosomal dominant polycystic kidney disease. Adv Chronic Kidney Dis 17:153-163

5. Ecder T, Chapman AB, Brosnahan GM et al (2000) Effect of antihypertensive therapy on renal function and urinary albumin excretion in hypertensive patients with autosomal dominant polycystic kidney disease. Am J Kidney Dis 35:427-432

6. Ecder T, Edelstein CL, Fick-Brosnahan GM et al (2001) Diuretics versus angiotensin-converting enzyme inhibitors in autosomal dominant polycystic kidney disease. Am J Nephrol 21:98-103

7. Ecder T, Schrier RW (2009) Cardiovascular abnormalities in autosomal-dominant polycystic kidney disease. Nat Rev Nephrol 5:221-228

8. Ecder T, Schrier RW (2001) Hypertension in autosomal-dominant polycystic kidney disease: early occurrence and unique aspects. J Am Soc Nephrol 12:194-200

9. Grantham JJ (2008) Clinical practice. Autosomal dominant polycystic kidney disease. N Engl J Med 359:1477-1485

10. Harris PC, Torres VE (2009) Polycystic kidney disease. Annu Rev Med 60:321-337

11. Jafar TH, Stark PC, Schmid CH et al (2005) The effect of angiotensin-converting-enzyme inhibitors on progression of advanced polycystic kidney disease. Kidney Int 67:265-271

12. Kanno Y, Suzuki H, Okada H et al (1996) Calcium channel blockers versus ACE inhibitors as antihypertensives in polycystic kidney disease. QJM 89:65-70 
13. Keith DS, Torres VE, Johnson CM et al (1994) Effect of sodium chloride, enalapril, and losartan on the development of polycystic kidney disease in Han:SPRD rats. Am J Kidney Dis 24:491-498

14. Kelleher CL, Mcfann KK, Johnson AM et al (2004) Characteristics of hypertension in young adults with autosomal dominant polycystic kidney disease compared with the general U.S. population. Am J Hypertens 17:1029-1034

15. Klahr S, Breyer JA, Beck GJ et al (1995) Dietary protein restriction, blood pressure control, and the progression of polycystic kidney disease. Modification of Diet in Renal Disease Study Group. J Am Soc Nephrol 5:2037-2047

16. Klein IH, Ligtenberg G, Oey PL et al (2001) Sympathetic activity is increased in polycystic kidney disease and is associated with hypertension. J Am Soc Nephrol $12: 2427-2433$

17. Mitobe M, Yoshida T, Sugiura H et al (2010) Clinical effects of calcium channel blockers and renin-angiotensin-aldosterone system inhibitors on changes in the estimated glomerular filtration rate in patients with polycystic kidney disease. Clin Exp Nephrol 14:573-577

18. Nutahara K, Higashihara E, Horie S et al (2005) Calcium channel blocker versus angiotensin II receptor blocker in autosomal dominant polycystic kidney disease. Nephron Clin Pract 99:c18-c23

19. Pirson Y (2010) Extrarenal manifestations of autosomal dominant polycystic kidney disease. Adv Chronic Kidney Dis 17:173-180

20. Rahbari-Oskoui F, Williams O, Chapman A (2014) Mechanisms and management of hypertension in autosomal dominant polycystic kidney disease. Nephrol Dial Transplant 29:2194-2201

21. Rizk D, Chapman AB (2003) Cystic and inherited kidney diseases. Am J Kidney Dis 42:1305-1317

22. Schrier R, Mcfann K, Johnson A et al (2002) Cardiac and renal effects of standard versus rigorous blood pressure control in autosomal-dominant polycystic kidney disease: results of a seven-year prospective randomized study. J Am Soc Nephrol 13:1733-1739

23. Schrier RW (2006) Optimal care of autosomal dominant polycystic kidney disease patients. Nephrology (Carlton) 11:124-130 
24. Schrier RW (2009) Renal volume, renin-angiotensin-aldosterone system, hypertension, and left ventricular hypertrophy in patients with autosomal dominant polycystic kidney disease. J Am Soc Nephrol 20:1888-1893

25. Schrier RW, Abebe KZ, Perrone RD et al (2014) Blood pressure in early autosomal dominant polycystic kidney disease. N Engl J Med 371:2255-2266

26. Schrier RW, Johnson AM, Mcfann K et al (2003) The role of parental hypertension in the frequency and age of diagnosis of hypertension in offspring with autosomal-dominant polycystic kidney disease. Kidney Int 64:1792-1799

27. Torres VE, Abebe KZ, Chapman AB et al (2014) Angiotensin blockade in late autosomal dominant polycystic kidney disease. N Engl J Med 371:2267-2276

28. Torres VE, Harris PC, Pirson Y (2007) Autosomal dominant polycystic kidney disease. Lancet 369:1287-1301

29. Van Dijk MA, Breuning MH, Duiser R et al (2003) No effect of enalapril on progression in autosomal dominant polycystic kidney disease. Nephrol Dial Transplant $18: 2314-2320$

30. Zafar I, Tao Y, Falk S et al (2007) Effect of statin and angiotensin-converting enzyme inhibition on structural and hemodynamic alterations in autosomal dominant polycystic kidney disease model. Am J Physiol Renal Physiol 293:F854-F859 
Tab. 1 Studienresultate der HALT-PKD-Studien. (Aus [25, 27])

\begin{tabular}{|c|c|c|}
\hline & Studie A & Studie B \\
\hline Anzahl Studienteilnehmer & 558 & 486 \\
\hline Alterskategorie & 15-49 Jahre & 18-64 Jahre \\
\hline GFR & $>60 \mathrm{ml} / \mathrm{min} / 1,73 \mathrm{~m}^{2}$ & $25-60 \mathrm{ml} / \mathrm{min} / 1,73 \mathrm{~m}^{2}$ \\
\hline Studienarme & $\begin{array}{l}\text { Standard (120-130/70-80 mmHg) vs. tiefer (95-110/ } \\
110 / 75 \mathrm{mmHg}) \mathrm{BD} \\
\text { Lisinopril + Placebo (L/P) vs. Lisinopril + Telmisartan } \\
\text { (L/T) }\end{array}$ & $\begin{array}{l}\text { Lisinopril + Placebo vs. Lisinopril + Tel- } \\
\text { misartan }\end{array}$ \\
\hline Primärer Endpunkt & $\begin{array}{l}\text { Vermindertes Zystenwachstum bei tieferem BD: } \\
\Delta \text { TKV } 5,6 \% \text { vs. } 6,6 \% \\
\text { Ähnliches Zystenwachstum bei L/P und L/T: } \\
\Delta \text { TKV } 6,2 \% \text { vs. } 6,0 \%\end{array}$ & $\begin{array}{l}\text { 50\%-Reduktion GFR, ESRD oder Tod } \\
\text { ähnlich (HR: } 1,08, \mathrm{KI}: 0,82-1,42 \text { ) }\end{array}$ \\
\hline Sekundäre Endpunkte & $\begin{array}{l}\text { Stärkere Reduktion des LVMI und der Albuminurie } \\
\text { bei tieferem BD, kein Unterschied bezüglich eGFR- } \\
\text { Verlust } \\
\text { Keine Unterschiede zwischen L/P und L/T bezüglich } \\
\text { LVMI, Albuminurie und eGFR-Verlust }\end{array}$ & $\begin{array}{l}\text { Kein Unterschied bezüglich eGFR-Verlust } \\
\text { und Albuminurie }\end{array}$ \\
\hline Nebenwirkungen & Schwindel und Taumel häufiger bei tieferem BD & \\
\hline
\end{tabular}




\begin{tabular}{|l|l|l|}
\hline & Studie A & Studie B \\
\hline & Keine Unterschiede zwischen L/P und L/T & Kein Unterschied bezüglich Hyperkaliämie \\
& & und akutem Nierenversagen \\
\hline
\end{tabular}

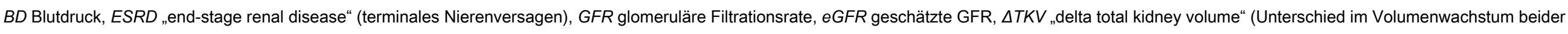

Nieren pro Jahr in Prozent), HR Hazard-Ratio KI 95\%-Konfidenzintervall, LVMI linksventrikulärer Massenindex. 